\title{
The Association of Cardio-Ankle Vascular Index and Ankle-Brachial Index in Patients with Peripheral Arterial Disease
}

\author{
Taichiro Hayase \\ Department of Cardiology, Yokohamashintoshi Neurosurgical Hospital, Yokohama, Japan
}

\section{Keywords}

Atherosclerosis · Arterial stiffness · Ankle-brachial blood pressure index . Cardio-ankle vascular index

\begin{abstract}
Introduction: The cardio-ankle vascular index (CAVI) is a well-known index to evaluate arterial stiffness and predict cardiovascular risk. Methods: We investigated whether CAVI can predict severity and extent of peripheral arterial disease. This study was a single-center, retrospective, observational study approved by the Ethics Committee of Yokohama Shintoshi Neurosurgical Hospital. A total of 96 patients (males, 63) with an abnormal ankle-brachial blood pressure index $(\mathrm{ABI})$ of $<0.9$ and who underwent extremity arteriography at our hospital from 2015 to 2018 were enrolled in this study. We defined that CAVI with a range of $<8.0$ was normal. $\boldsymbol{R e}$ sults: Coronary angiography and extremity arteriography were performed for patients who had intermittent claudication and abnormal $A B I$. We divided the affected limbs into 3 categories: above-the-knee artery stenosis, above-the-knee artery chronic total occlusion, and only below-the-knee artery stenosis/occlusion groups. CAVI pseudonormalization was seen in 28,76 , and $19 \%$, respectively. The above-the-
\end{abstract} nosis/occlusion groups had a high odds ratio of abnormalization of CAVI (3.1, 95\% confidence interval [CI]: 1.39-7.22; $p=0.05$, 4.56, 95\% Cl: 1.64-14.7). Discussion/Conclusion: In the presence of the above-the-knee artery chronic total occlusion, CAVI pseudonormalization was likely to be seen. The presence of CTO in the above-the-knee artery is one cause of pseudonormalized CAVI. In the range of $\mathrm{ABI}$, in which stenotic lesions and obstructive lesions coexist, it may be possible to detect the existence of CTO by a combination of both $A B I$ and CAVI.

(c) 2021 S. Karger AG, Basel

\section{Introduction}

Atherosclerosis is a major contributor to the development of cerebrocardiovascular diseases and is a major cause of mortality and morbidity [1]. It is well known that hypertension, diabetes mellitus, smoking, and dyslipidemia play a major role in the development of atherosclerosis [2]. Many clinicians have attempted to assess the extent of atherosclerosis using some surrogate vascular measurements for patients with multiple risk factors. 
Pulse wave velocity (PWV) is the gold standard measurement of arterial stiffness [3], and the ankle-brachial blood pressure index (ABI) is a simple and widely used clinical test for the assessment of peripheral arterial disease (PAD) [4-7].

PAD is caused by atherosclerosis. Patients with PAD have a higher prevalence of atherosclerosis in the coronary, carotid, and renal arteries than in patients without PAD [8]. PAD is widely known as a predictor of future cardiovascular and cerebrovascular morbidity and mortality $[9,10]$. A resting $\mathrm{ABI}$ is recommended to establish a diagnosis of PAD. PWV is the gold standard measurement of arterial stiffness; however, there are several limitations with this method, chiefly fluctuations in blood pressure [11], and abnormal values of ABI lead to the pseudonormalization of PWV. CAVI has been widely accepted since its introduction in 2004 and applied to a variety of studies, specifically those involving the evaluation of arterial stiffness in patients with cardiovascular diseases [12-19]. Although CAVI has been extensively studied and recognized as a tool to predict cardiovascular risk [20-22], there has been little research on the relation of $\mathrm{ABI}$ and CAVI for patients with PAD. In particular, previous studies did not examine differences in lesions (stenosis or obstruction) due to differences in whether CAVI was normal or not when ABI decreased. The purpose of this study was to investigate whether CAVI can predict the severity and extent of PAD in the same manner as ABI.

\section{Methods}

\section{Study Design and Patients}

This was a single-center, retrospective, observational chart review approved by the Ethics Committee of Yokohama Shintoshi Neurosurgical Hospital and conducted from 2015 to 2018. The study has therefore been performed in accordance with the ethical standards laid down in the 1964 Declaration of Helsinki and its later amendments. Patients and their families were informed about the purpose and process of this study, and they agreed and signed informed consent. Patients with an abnormal ABI of $<0.9$ who underwent extremity arteriography and visited the hospital from 2015 to 2018 were included in this study. Patients with severe renal dysfunction were excluded because there is no medical equipment such as a dialysis apparatus to treat patients with chronic kidney disease.

\section{Hypertension}

Hypertension was defined as receiving antihypertensive medication or if the average systolic or diastolic blood pressure measured was $>140$ or $90 \mathrm{~mm} \mathrm{Hg}$, respectively, during at least 2 different occasions.
Table 1. Baseline characteristics

\begin{tabular}{lc}
\hline Variables & $\begin{array}{l}\text { Overall } \\
95(190 \text { limbs })\end{array}$ \\
\hline Age, years & $73 \pm 8$ \\
Female sex & $32(34)$ \\
ABI < 0.9 & 132 limbs \\
Coronary angiography & \\
0-vessel disease & $21(22)$ \\
1-vessel disease & $31(32)$ \\
2-vessel disease & $22(23)$ \\
3-vessel disease & $21(22)$ \\
Past medical history & \\
Hypertension & $81(85)$ \\
Dyslipidemia & $60(63)$ \\
Hyperuricemia & $14(14)$ \\
Diabetes mellitus & $36(37)$ \\
Atrial fibrillation & $23(24)$ \\
Cardiovascular disease & $24(25)$ \\
Cerebral vascular disease & $71(74)$ \\
Medication & \\
Antiplatelet agents & $95(100)$ \\
ACEI/ARB & $51(53)$ \\
B-Blocker & $31(32)$ \\
Ca-Blocker & $62(65)$ \\
Statin & $51(53)$ \\
Insulin & $9(9)$ \\
& \\
&
\end{tabular}

Dyslipidemia

Dyslipidemia was defined as an LDL-cholesterol of $>140 \mathrm{mg} /$ $\mathrm{dL}$ or an HDL-cholesterol of $<40 \mathrm{mg} / \mathrm{dL}$ or triglyceride levels $>150$ $\mathrm{mg} / \mathrm{dL}$ or documented hypercholesterolemia requiring lipid-lowering drug therapy.

\section{Hyperuricemia}

Hyperuricemia was defined as urate concentrations exceeding about $7 \mathrm{mg} / \mathrm{dL}$ or therapeutic drug administration.

\section{Diabetes Mellitus}

Diabetes mellitus was defined as present if the patient had a history of diabetes, diabetic therapeutic drug administration, or if fasting plasma glucose exceeded 126 or $200 \mathrm{mg} / \mathrm{dL}$ at any time.

\section{Vascular Screening}

CAVI was determined by using a small-sized vascular screening system (VS1500AE; Fukuda Denshi Co., Ltd., Tokyo Japan), which is available in most clinics in Japan $[23,24]$. A CAVI value $<8$ was interpreted as a mild risk of arteriosclerosis, that between 8 and 9 was interpreted as a moderate risk of arteriosclerosis, and that $>9$ was interpreted as a high risk of arteriosclerosis [25]. In this study, $\mathrm{CAVI} \geq 9.0$ was defined as abnormal. ABI was simultaneously measured using the same equipment (VS1500AE). ABI was used to screen for $\mathrm{PAD}$, and subjects were divided into 4 groups according to $\mathrm{ABI}$ (high ankle pressure group, $>1.40$; normal group, $\leq 1.00$ but $\leq 1.40$; normal but borderline group, $<0.90$ but $<1.00$; and suspected ASO group, $\leq 0.90$ ) [20]. Coronary angiography and extremity
Hayase 
Table 2. The comparison of extremity arteriography findings according to the CAVI

\begin{tabular}{lccc}
\hline & CAVI $<9$ & CAVI $\geq 9$ & $p$ value \\
\hline$N$ & 59 & 73 & \\
ABI & $0.61 \pm 0.15$ & $0.77 \pm 0.10$ & $<0.01$ \\
Site of the lesion & & & \\
$\quad$ Common iliac artery stenosis & $18(30)$ & $10(13)$ & 0.031 \\
$\quad$ External iliac artery stenosis & $13(22)$ & $10(13)$ & 0.252 \\
$\quad$ Femoral artery stenosis & $6(10)$ & $2(2)$ & 0.139 \\
$\quad$ Superficial femoral artery stenosis & $40(67)$ & $31(42)$ & 0.005 \\
$\quad$ Popliteal artery stenosis & $3(5)$ & $1(1)$ & 0.324 \\
$\quad$ Only below-the-knee artery stenosis & $6(10)$ & $25(34)$ & 0.004 \\
\hline
\end{tabular}

Table 3. The comparison of CAVI and ABI in 3 groups (aboveknee artery stenosis, above-knee artery chronic complete occlusion, and above-knee artery stenosis/occlusion only groups)

\begin{tabular}{lccc}
\hline & $\begin{array}{l}\text { Above-the-knee } \\
\text { artery stenosis } \\
\text { group }\end{array}$ & $\begin{array}{l}\text { Above-the-knee } \\
\text { artery chronic total } \\
\text { occlusion group }\end{array}$ & $\begin{array}{l}\text { Only below- } \\
\text { the-knee artery } \\
\text { stenosis group }\end{array}$ \\
\hline$N$ & 50 & 51 & 31 \\
ABI & $0.77 \pm 0.09$ & $0.59 \pm 0.11$ & $0.82 \pm 0.11$ \\
CAVI $<9$ & $14(28)$ & $39(76)$ & $6(19)$ \\
CAVI $\geq 9$ & $36(72)$ & $12(24)$ & $25(81)$ \\
\hline
\end{tabular}

arteriography were performed within a month for patients who had intermittent claudication and abnormal ABI. The relation among coronary artery diseases and peripheral artery disease and the patients' background (risk factors, CAVI, and ABI) were investigated.

\section{Statistical Analysis}

Statistical analyses were performed using EZR, which is a graphical user interface for $\mathrm{R}$ based on $\mathrm{R}$ commander. More precisely, it is a modified version of $\mathrm{R}$ commander designed to add statistical functions frequently used in biostatistics [26]. Fisher's exact test and Mann-Whitney U test were performed to determine whether intragroup differences were statistically significant. Logistic regression analysis was used to identify the predictors of abnormal CAVI and expressed as odds ratio with $95 \%$ confidence interval. Data are presented as mean \pm standard deviation or number or percentage. In all statistical procedures, 2 -sided $p$ values $<0.05$ were considered statistically significant.

\section{Result}

\section{Characteristics of the Study Population}

The baseline characteristics of our study are shown in Table 1 . The median age was 73 years, and $32 \%$ of the patients included in the study were female. Seventy-seven percent of patients had a history of acute stroke and were scrutinized during the chronic phase after treatment. The others were admitted in our hospital by screening for cardiovascular disease. Ninety-five patients (132 limbs) had a low ABI. All patients had been prescribed antiplatelet agents. Angiotensin-converting enzyme inhibitors or angiotensin receptor blockers, $\beta$-blockers, calcium channel blockers, and statins were prescribed for $53,32,65$, and $53 \%$ of patients, respectively. Coronary artery disease was evaluated by coronary angiography: $22,32,23$, and $22 \%$ of patients were 0 vessel disease (0VD), 1VD, 2VD, and 3VD, respectively.

\section{Comparison of Extremity Arteriography Findings according to the CAVI}

In total, 95 patients (132 limbs) had a low ABI. The affected limbs were stratified into 2 groups as follows: 59 limbs in the normal CAVI group (CAVI $<9$ ), while 73 limbs in the abnormal CAVI group (CAVI $\geq 9$ ) (Table 2). The normal CAVI group had significantly lower ABI, and therefore the prevalence of common iliac artery stenosis and superficial femoral artery stenosis was significantly higher in the normal CAVI group ( $p=0.031$ and 0.005 , respectively) Next, we divided the affected limbs into 3 categories: the above-the-knee artery stenosis, above-theknee artery chronic total occlusion, and only below-theknee artery stenosis/occlusion groups (Tables 3-5). CAVI pseudonormalization was seen in 28,76 , and $19 \%$, respectively (Table 3). The above-the-knee artery stenosis and only below-the-knee artery stenosis/occlusion had a high odds ratio (OR) with an abnormal CAVI (3.1, 95\% CI: 1.39-7.22; $p=0.05,4.56$, 95\% CI: 1.64-14.7) (Tables 4, 5). CAVI pseudonormalization was likely to be seen in the group with above-the-knee artery chronic total occlusion. CAVI showed differences in the group with abovethe-knee artery chronic total occlusion when ABI was between 0.7 and $0.9(p=0.02)$ (Table 6). The group with above-the-knee artery chronic total occlusion had a higher OR of CAVI pseudonormalization (5.22, 95\% CI: 1.2621.6; $p=0.02)($ Table 7$)$. 
Table 4. The comparison of extremity arteriography findings according to the CAVI

Table 5. The lesion characteristic to predict the abnormalized CAVI

\begin{tabular}{lccc}
\hline & CAVI $<9$ & CAVI $\geq 9$ & $p$ value \\
\hline$n$ & 59 & 73 & \\
ABI & $0.61 \pm 0.15$ & $0.77 \pm 0.10$ & $<0.01$ \\
Extremity arteriography & & & \\
$\quad$ Above-the-knee artery stenosis & $14(23)$ & $36(49)$ & $<0.01$ \\
$\quad$ Above-the-knee artery chronic total occlusion & $39(66)$ & $12(16)$ & $<0.01$ \\
$\quad$ Only below-the-knee artery stenosis & $6(10)$ & $25(34)$ & $<0.01$ \\
\hline
\end{tabular}

\begin{tabular}{llrr}
\hline Abnormalized CAVI & Odds ratio & $\begin{array}{l}\text { 95\% confidence } \\
\text { interval }\end{array}$ & $p$ value \\
\hline Above-the-knee artery stenosis & 3.1 & $1.39-7.22$ & 0.005 \\
Above-the-knee artery chronic total occlusion & 0.1 & $0.040-0.25$ & $<0.001$ \\
Only below-the-knee artery stenosis & 4.56 & $1.64-14.7$ & 0.002 \\
\hline
\end{tabular}

Table 6. The prevalence of chronic total occlusion when ABI was between 0.7 and $0.9(N=79)$

\begin{tabular}{llll}
\hline & CAVI $<9$ & CAVI $\geq 9$ & $p$ value \\
\hline$N$ & 31 & 48 & \\
$\begin{array}{l}\text { Extremity arteriography } \\
\text { Above-the-knee artery chronic total occlusion }\end{array}$ & $8(25.8)$ & $3(6.3)$ & 0.02 \\
\hline
\end{tabular}

Table 7. Pseudonormalized CAVI predicts CTO when ABI was between 0.7 and 0.9

\begin{tabular}{llll}
\hline Pseudonormalized CAVI & Odds ratio & $\begin{array}{l}\text { 95\% confidence } \\
\text { interval }\end{array}$ & $p$ value \\
\hline Above-the-knee artery chronic total occlusion & 5.22 & $1.26-21.56$ & $<0.02$ \\
\hline
\end{tabular}

\section{Discussion}

PWV is the gold standard measurement of arterial stiffness; however, there are several limitations of this method, chiefly fluctuations in blood pressure [11]. Since CAVI is less susceptible to fluctuations in blood pressure than PWV, it has attracted attention as the most reliable indicator of arteriosclerosis. A recent study on acute coronary syndrome patients reported high MACE in the high CAVI group (CAVI $\geq 8.35$ ) [27]. CAVI has been extensively studied and recognized as a tool to predict cardiovascular risk $[11,19-21,28]$, but it is known that abnormal values of ABI lead to pseudonormalization of PWV; there is a similar report for CAVI. In the previous studies, patients with $\mathrm{ABI}<0.9$ were excluded because of a possibility of falsely low CAVI and PWV. Therefore, almost no studies have been conducted in patients with a high risk of cerebral cardiovascular events, such as polyvascular disease patients in this study [29] (Table 1). In
PAD patients, ABI decreases and CAVI pseudonormalizes as the lesion progresses. In this study, the ABI was significantly lower in the normal CAVI group than in the abnormal CAVI group. Further, the prevalence of common iliac artery stenosis and superficial femoral artery stenosis was significantly higher in the normal CAVI group than in the abnormal CAVI group (Table 2). In case CAVI was falsely pseudonormalized, the abnormal CAVI group may not have significantly higher rates of major cerebral cardiovascular events than the normal CAVI group. Thus, care should be taken while interpreting CAVI values in PAD patients.

This study examined the relation of CAVI and stenosis or occlusion of the lower limb arteriography in patients with low ABI (Tables 4, 5). One explanation for the mechanism of CAVI pseudonormalization might be as follows: as atherosclerosis progresses, the PWV propagating the blood vessels will be faster. CAVI also will be higher as stenosis progresses. However, when the stenotic lesion 
Fig. 1. Differentiation method of stenotic/ occluded lesions using CAVI when ABI is low.

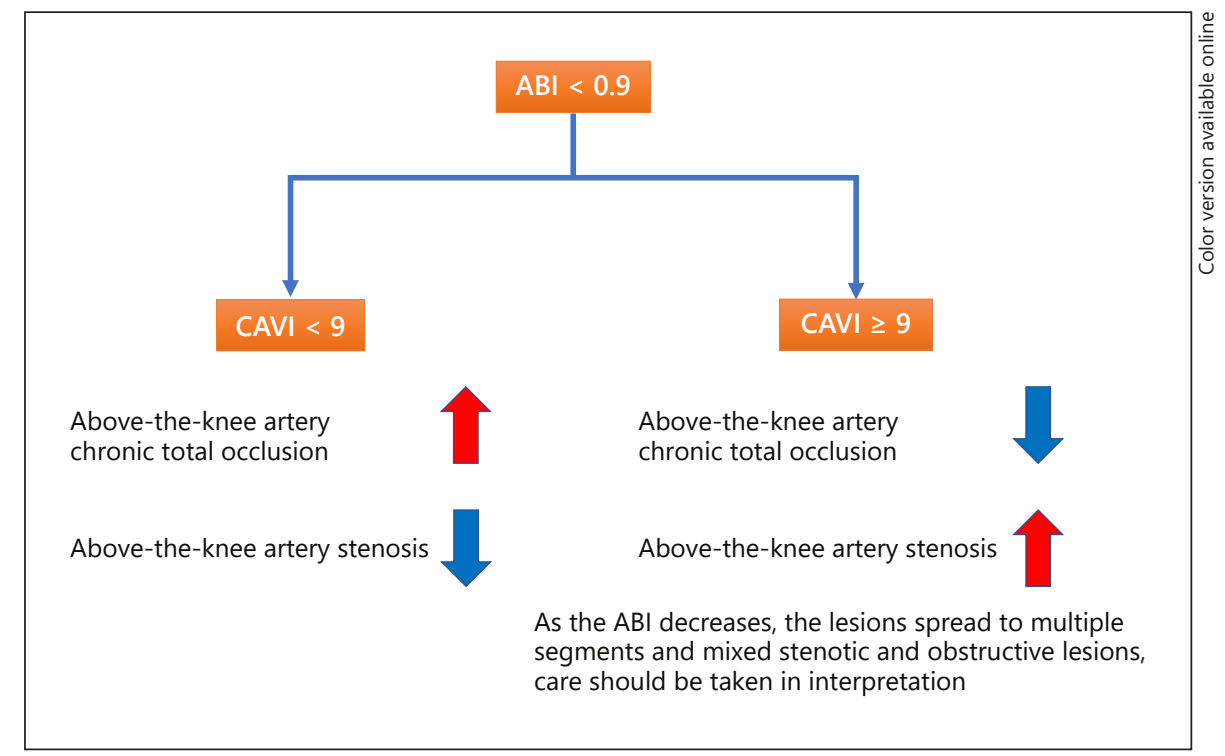

further progresses to obstruction, the blood flow will pass through the formed collateral circulation and the flow velocity will be slow, so that it may be that the CAVI will be falsely normalized. This is only a hypothesis because the process from stenosis to occlusion and collateral circulation formation cannot be directly observed in lower limb lesions, and the influence of stenosis or occlusion of the below-knee lesions has not been investigated because it is difficult to perform detailed examination due to poor angiography. This point was considered to be the limitation of this study.

Previous research showed that if the ABI is $<0.90$, occlusive arterial disease may be present. If a value of $<0.80$ is noted, it is highly likely that vascular disease will be found. ABIs between 0.50 and 0.80 are likely to be found in patients with single-segment occlusions, while ABIs $<0.50$ are commonly found in patients with multisegment disease $[30,31]$. It is conceivable that the lower the ABI, the higher the prevalence of CTO in the lower limbs. However, there is no report that the presence of CTO in the above-knee artery can be diagnosed only from the ABI value. The present study showed that CAVI tended to be abnormal in stenotic lesions and pseudonormalized in obstructive lesions in patients with PAD (Table 5). Particularly, in the range of $\mathrm{ABI}$, where stenotic lesions and obstructive lesions may coexist, the above-the-knee artery chronic total occlusion group had a higher OR of pseudonormalization of CAVI (5.22, 95\% CI: 1.26-21.6; $p=0.02$ ) (Tables 6,7$)$. It is noteworthy and useful that it may be possible to detect the existence of CTO by a combination of both ABI and pseudonormalized CAVI be- cause we can simultaneously measure ABI and CAVI, which are noninvasive methods (Fig. 1).

\section{Study Limitation}

There are several limitations to this study. First, it was single-center, retrospective study with a small sample size. Second, since patients with renal dysfunction have been excluded, maintenance dialysis patients and CKD patients who are more likely to have severe calcification lesions have not been studied. Also, many patients in this study were polyvascular disease patients with advanced arteriosclerosis, and it may be difficult to generalize results to a wider population. Third, since patients with critical limb ischemia were not included, angiography for the below-the-knee artery was not examined in detail. This might influence our results.

\section{Conclusion}

CAVI may be used to detect the existence of CTO and to predict the severity and extent of PAD.

\section{Statement of Ethics}

All procedures performed in studies involving human participants were in accordance with the ethical standards of the institutional and/or national research committee and 1964 Helsinki Declaration and its later amendments or comparable ethical standards. All participating patients provided written informed consent. The study protocol was reviewed and approved by institutional review boards and ethics committee of our hospital. 


\section{Conflict of Interest Statement}

The authors have no conflicts of interest to declare.

\section{Funding Sources}

The authors did not receive any funding.

\section{References}

1 Greenland P, Smith SC Jr, Grundy SM. Improving coronary heart disease risk assessment in asymptomatic people: Role of traditional risk factors and noninvasive cardiovascular tests. Circulation. 2001;104:1863-7.

2 Ankle Brachial Index Collaboration; Fowkes FG, Fowkes FG, Murray GD, Butcher I, Heald CL, Lee RJ, et al. Ankle brachial index combined with Framingham Risk Score to predict cardiovascular events and mortality: a metaanalysis. JAMA. 2008;300:197-208.

3 Tomiyama H, Matsumoto C, Shiina K, Yamashina A. Brachial-Ankle PWV: current status and future directions as a useful marker in the management of cardiovascular disease and/or cardiovascular risk factors. J Atheroscler Thromb. 2016;23:128-46.

4 Martyn CN, Gale CR, Jespersen S, Sherriff SB. Impaired fetal growth and atherosclerosis of carotid and peripheral arteries. Lancet. 1998; 352:173-8.

5 Busch MA, Lutz K, Röhl JE, Neuner B, Masuhr F. Low ankle-brachial index predicts cardiovascular risk after acute ischemic stroke or transient ischemic attack. Stroke. 2009;40: $3700-5$.

6 Kajikawa M, Maruhashi T, Iwamoto Y, Iwamoto A, Matsumoto T, Hidaka T, et al. Borderline ankle-brachial index value of 0.910.99 is associated with endothelial dysfunction. Circ J. 2014;78:1740-5.

7 Gerhard-Herman MD, Gornik HL, Barrett C, Barshes NR, Corriere MA, Drachman DE, et al. 2016 AHA/ACC Guideline on the management of patients with lower extremity peripheral artery disease: a report of the American College of Cardiology/American Heart Association Task Force on Clinical Practice Guidelines. Circulation. 2017;135:e726-79.

8 Gerhard-Herman MD, Gornik HL, Barrett C, Barshes NR, Corriere MA, Drachman DE, et al. 2016 AHA/ACC guideline on the management of patients with lower extremity peripheral artery disease: a report of the American College of Cardiology/American Heart Association Task Force on Clinical Practice Guidelines. Circulation. 2017;135:e726-79.

9 US Centers for Disease Control and Prevention [updated 2013 May 8; cited 2014 January 11]. Available from: https://www.cdc.gov/.

10 Sun CK. Cardio-ankle vascular index (CAVI) as an indicator of arterial stiffness. Integr Blood Press Control. 2013;6:27-38.
11 Nakamura K, Tomaru T, Yamamura S, Miyashita Y, Shirai K, Noike H. Cardio-ankle vascular index is a candidate predictor of coronary atherosclerosis. Circ J. 2008;72:598604.

12 Saiki A, Sato Y, Watanabe R, Watanabe Y, Imamura $\mathrm{H}$, Yamaguchi $\mathrm{T}$, et al. The role of a novel arterial stiffness parameter, Cardio-Ankle Vascular Index (CAVI), as a surrogate marker for cardiovascular diseases. J Atheroscler Thromb. 2016;23:155-68.

13 Takaki A, Ogawa H, Wakeyama T, Iwami T, Kimura M, Hadano Y, et al. Cardio-ankle vascular index is superior to brachial-ankle pulse wave velocity as an index of arterial stiffness. Hypertens Res. 2008;31:1347-55.

14 Shirai K. Analysis of vascular function using the cardio-ankle vascular index (CAVI). Hypertens Res. 2011;34:684-5.

15 Horinaka S, Yabe A, Yagi H, Ishimura K, Hara $\mathrm{H}$, Iemura $\mathrm{T}$, et al. Cardio-ankle vascular index could reflect plaque burden in the coronary artery. Angiology. 2011;62:401-8.

16 Saji N, Kimura K, Shimizu H, Kita Y. Silent brain infarct is independently associated with arterial stiffness indicated by cardio-ankle vascular index (CAVI). Hypertens Res. 2012; 35:756-60.

17 Asmar R. Principles and usefulness of the cardio-ankle vascular index (CAVI): a new global arterial stiffness index. Eur Heart J. 2017; 19(Suppl 1_B):B4-10.

18 Ato D. Brachial-ankle pulse wave velocity, cardio-ankle vascular index, and prognosis. Vasc Health Risk Manag. 2018;14:321-48.

19 Alpert JS. Cardiology patient page: What you need to know if you have coronary artery disease. Circulation. 2011;124:e176-e178.

20 Sato Y, Nagayama D, Saiki A, Watanabe R, Watanabe Y, Imamura $\mathrm{H}$, et al. Cardio-ankle vascular index is independently associated with future cardiovascular events in outpatients with metabolic disorders. J Atheroscler Thromb. 2016;23:596-605.

21 Park JB, Park HE, Choi SY, Kim MK, Oh BH Relation between cardio-ankle vascular index and coronary artery calcification or stenosis in asymptomatic subjects. J Atheroscler Thromb. 2013;20:557-67.
$22 \mathrm{Hu} \mathrm{H}$, Cui H, Han W, Ye L, Qiu W, Yang H, et al. A cutoff point for arterial stiffness using the cardio-ankle vascular index based on carotid arteriosclerosis. Hypertens Res. 2013;36: $334-41$.

23 Nishizawa Y, Shoji T, Maekawa K, Nagasue K, Okuno S, Kim M, et al. Intima-media thickness of carotid artery predicts cardiovascular mortality in hemodialysis patients. Am J Kidney Dis. 2003;41:S76-9.

24 Shirai K, Utino J, Otsuka K, Takata M. A novel blood pressure-independent arterial wall stiffness parameter; cardio-ankle vascular index (CAVI). J Atheroscler Thromb. 2006;13: 101-7.

25 Gerhard-Herman MD, Gornik HL, Barrett C, Barshes NR, Corriere MA, Drachman DE, et al. 2016 AHA/ACC Guideline on the management of patients with lower extremity peripheral artery disease: executive summary: a report of the American College of Cardiology/ American Heart Association Task Force on Clinical Practice Guidelines. Circulation. 2017;135:e686-725.

26 Srinubab G, Allam AR, Narashima RM. Identification of biomarkers for type 2 diabetes and its complications: a bioinformatic approach. Int J Biomed Sci. 2007;3:229-36.

27 Kanda Y. Investigation of the freely available easy-to-use software "EZR" for medical statistics. Bone Marrow Transplant. 2013;48:452-8.

28 Kirigaya J, Iwahashi N, Tahakashi H, Minamimoto Y, Gohbara M, Abe T, et al. Impact of cardio-ankle vascular index on long-term outcome in patients with acute coronary syndrome. Atheroscler Thromb. 2019;26:15409.

29 Park JB, Park HE, Choi SY, Kim MK, Oh BH Relation between cardio-ankle vascular index and coronary artery calcification or stenosis in asymptomatic subjects. J Atheroscler Thromb. 2013;20:557-67.

30 Bhatt DL, Eagle KA, Ohman EM, Hirsch AT, Goto S, Mahoney EM, et al. Comparative determinants of 4-year cardiovascular event rates in stable outpatients at risk of or with atherothrombosis. JAMA. 2010;304:1350-7.

31 Orchard TJ, Strandness DE Jr. Assessment of peripheral vascular disease in diabetes. Report and recommendations of an international workshop sponsored by the American Diabetes Association and the American Heart Association September 18-20, 1992 New Orleans, Louisiana. Circulation. 1993;88:819-28. 